\title{
MALDI imaging as a specific diagnostic tool for routine cervical cytology specimens
}

\author{
KRISTINA SCHWAMBORN ${ }^{1}$, RENÉ C. KRIEG ${ }^{1 *}$, SANDRA UHLIG ${ }^{1}$, \\ HANS IKENBERG $^{2}$ and AXEL WELLMANN ${ }^{3}$ \\ ${ }^{1}$ Institute of Pathology, RWTH Aachen University, Aachen; ${ }^{2}$ CytoMol, Frankfurt; \\ ${ }^{3}$ Institute of Pathology, Celle, Germany
}

Received September 22, 2010; Accepted November 15, 2010

DOI: $10.3892 / \mathrm{ijmm} .2010 .587$

\begin{abstract}
Cervical squamous cell carcinoma (SCC) is among the most common malignancies in women worldwide. In developed countries routine cytology screening has dramatically reduced SCC mortality within the last three decades. High risk (HR) human papilloma virus (HPV) infection is the main causal factor in nearly $100 \%$ of invasive SCCs, in most cases of low grade squamous intraepithelial lesion (LSIL) and in nearly all cases of high grade squamous intraepithelial lesion (HSIL). Detection of HR-HPV DNA has been extensively evaluated for the triage of patients with low grade cytological abnormalities in order to identify those at greatest risk for underlying or developing HSIL or SCC. However, the vast majority of HR-HPV-positive precursor lesions will not progress to invasive cancer. A variety of other screening tools are available which aim to stratify clinically significant HPV infections and cytological alterations. Matrix assisted laser desorption/ionization (MALDI) imaging mass spectrometry is a promising technology to assist in this endeavor. It delivers accurate mass spectrometric information of the sample's proteins and enables the visualization of the spatial distribution of protein expression profiles in correlation with histological features. In this study, 18 samples with Pap IIID or higher (>LSIL) and 14 samples with Pap I-II (WNL) were analyzed by MALDI imaging mass spectrometry (IMS). A genetic algorithm was applied to classify spectra resulting in an overall cross validation, sensitivity for Pap IIID and Pap I-II of $83.7 \%, 88.9 \%$ and $78.6 \%$, respectively. As this IMS based approach allows for unbiased and automated classification of cytological samples it appears to be a promising tool for stratification of cervical Pap smears.
\end{abstract}

Correspondence to: Professor Axel Wellmann, Institute of Pathology, Wittinger Strasse 14, D-29223 Celle, Germany

E-mail: axel_wellmann@yahoo.com

"Dedicated to a friend who died far too sudden and early

Key words: cervical cytology, matrix assisted laser desorption/ ionization imaging, proteomics, human papilloma virus, diagnostics, screening, pathology

\section{Introduction}

Cervical squamous cell carcinoma (SCC) is the second most common cancer in women worldwide. In industrialized nations, however, cervical neoplasia ranks much lower mainly due to the success of cervical cytology as a screening tool for the detection of its precursor lesions (1-3). In the United States and Europe millions of Pap smears are performed yearly (approximately 50 million Pap smears in the USA alone) (4). The Munich classification and the Bethesda system (TBS) are the most commonly used classification schemes, stratifying cytological findings as normal/benign, reactive changes, squamous cell abnormalities, and glandular cell abnormalities.

Although a significant portion of patients with low grade squamous cell abnormalities of the cervical smear has an underlying high grade squamous intraepithelial lesion (HSIL) on cervical biopsy, the majority of these patients do not have clinically significant lesions (5-7). However, essentially all patients with atypical cells of undetermined significance (ASCUS) or of a low grade squamous intraepithelial lesion (LSIL) on a Pap smear screening require further evaluation by repeated cytology or colposcopic examination to rule out underlying HSIL. Application of molecular adjunctive tests could provide enhanced and objective bases for the triage of patients with clinically significant disease. This would avoid unnecessary procedures and expenditures. HR (high risk)-HPV (human papilloma virus) infection is the main causal factor in the vast majority of invasive SCCs and also in most cases of LSIL and nearly all HSIL. In addition, up to $95 \%$ of endocervical adenocarcinomas are associated with HPV infection. Over 100 different HPV types have now been characterized based on nucleotide sequence differences of the viral genome, including over 40 infecting the cervical mucosa $(8,9)$. Epidemiologic studies have divided HPVs into 'low risk' (LR) types and 'high risk' types, according to their risk for malignant transformation (8). HPV oncoproteins, especially E6 and E7, are directly involved in the pathogenesis of cervical carcinoma by interaction with p53 and the $\mathrm{Rb}$ proteins, p107 and p130 (8-10). HPV DNA detection has been extensively evaluated for the triage of patients with low grade cytologic abnormalities in order to identify those at greatest risk for underlying HSIL or SCC. Data from studies, such as the ASCUS-LSIL Triage Studies have indicated that 
HPV detection alone was not useful for the triage of patients with LSIL due to the high rate of positivity in this cytologic subgroup. A positive test result did not reliably predict clinically significant disease as demonstrated by the presence of HPV in over a third of the cases that were negative for HSIL or SCC on colposcopically directed biopsies (11).

A variety of other screening tools are available to stratify clinically significant HPV infections and cytological alterations, such as p16. Its subsequent overexpression is caused by the expression of HPV oncogenes, regardless of the HPV type and indicates a transforming infection. With commercially available tests (e.g. CINtec ${ }^{\mathrm{TM}}$ ) p16 can be identified immunocytochemically in cytology preparations. Moreover, p16 staining can facilitate the identification of abnormal cells in cytology preparations and has been evaluated as a biomarker to triage equivocal and low grade cytological findings (12). Although there are many other biomarker candidates (e.g. Ki-67, MYC, cyclin), data on their clinical utility are still very limited (13).

Proteomics encompass numerous platform technologies to study the proteome. As the proteome is more complex and dynamic than the genome it can be used as a sensitive sensor for ongoing alterations $(14,15)$. One promising new technology for protein analysis from intact biological tissue specimens is MALDI (matrix assisted laser desorption/ionization) imaging mass spectrometry (IMS) (16). Tissue sections are coated with matrix and analyzed at regular spatial intervals using MALDI mass spectrometry $(17,18)$. Therefore, this method allows the visualization of the spatial distribution of proteins in tissue samples $(19,20)$.

The present IMS based study was performed to develop a pilot approach to screen routine cervical cytology specimen in an automated manner for cytological classification into reactive and dysplastic lesions, respectively. As cytology specimens remain intact throughout the IMS analysis comparison with the classical cytological evaluation for validation of questionable results is possible.

\section{Materials and methods}

Sample collection/patient specimen. Cervical cytology specimens were obtained from patients undergoing gynaecological examination with cervical cytology in the Department of Gynaecology, RWTH Aachen University and at CytoMol laboratory, Frankfurt, Germany. All samples were collected under standard protocols. Briefly, cervical scrapings were taken by cytobrush and immediately immersed in ThinPrep ${ }^{\circledR}$ medium (Hologic Inc., Bedford, MA). Cytology specimens were processed by the T2000 processor (Hologic Inc.), which semi-automatically transfers the cells as a thin layer to a glass slide. Slides were examined by a board certified cytologist and finally a board certified pathologist for Pap classification. Eighteen samples with Pap IIID or higher (TBS: > LSIL) and 14 samples with Pap I-II (TBS: WNL) were included in this study.

Ten cervical samples were recruited from routine uterine specimens for pilot study to demonstrate differential expression of defensin. All specimens were procured from patients giving informed consent and with the approval of the Ethics Commission.
Sample preparation and MS analysis. For IMS analysis, cytospin dilutions were prepared from each sample to achieve a thin cell layer. Indium tin oxide-coated conductive glass slides (Bruker Daltonics, Germany) were used for MS analysis. Slides were then washed in $70 \%$ ethanol (HPLC grade) for $30 \mathrm{sec}$ and in $96 \%$ ethanol (HPLC grade) for $15 \mathrm{sec}$. Samples were evenly spray-coated with matrix solution (180 mg sinapinic acid matrix (Bruker) in $5000 \mu 1$ LC-MS water, $5000 \mu \mathrm{l}$ acetonitrile and $10 \mu \mathrm{l}$ trifluoroacetic acid (all high pure reagents from Sigma) using a TLC (thin layer chromatography) spraying device (Sigma). Care was taken to standardize matrix application over the case number.

Imaging experiments were performed on a Reflex IV MALDI-TOF-MS (Bruker) and controlled by the FlexControl 3.0 software package (Bruker). Image acquisition of the spray coated cytology specimens was carried out using the FlexImaging 2.0 software (Bruker). A measuring array with a $200 \mu \mathrm{m}$ center to center spacing between each spot was chosen. At each measuring point 20 sufficient laser shots were averaged per spectrum.

Following imaging experiments the matrix was removed by incubating slides twice in methanol (100\%, room temperature, $5 \mathrm{~min}$ ) and once in acetone (100\%, room temperature, $5 \mathrm{~min})$. After air drying slides were Pap-stained to confirm diagnosis by a board certified cytologist.

Data analysis. Statistical analyses were performed using ClinProTools 2.2 software (Bruker) to distinguish between positive (Pap $\geq$ IIID) and negative (Pap I-II) cytology specimens. Therefore, spectra from representative regions from each sample were loaded into the software and grouped according to their diagnosis. Baseline correction was achieved using a top hat algorithm with a $10 \%$ minimal baseline width. Subsequently, all spectra were normalized to their own total ion count and calibrated. Classification models used in this study were built using a five dimensional genetic algorithm (GA) and a support vector machine algorithm (SVM), respectively. Additionally, the GA-based model was used to classify each spectrum of representative samples into one of the two groups (positive or negative cytology result). Exporting these data into FlexImaging enables the visualization of results by classifying the section in a color encoded depiction (class image).

Immunohistochemistry. Immunohistochemistry of tissue sections was performed as previously described (21). Briefly, paraffin-embedded cervical tissue sections were mounted on glass slides and deparaffinized in xylene. Antigen retrieval was achieved by 8 min digestion. Slides were permeabilized in a 1:1 acetone/methanol solution, washed, and incubated in $3 \%$ hydrogen peroxide. Sections were incubated with $\alpha$-defensin (DEFA) antibody (AbD Serotec) diluted 1:300 for $1 \mathrm{~h}$ at $25^{\circ} \mathrm{C}$ followed by exposure to an ultraView DAB kit protocol (Ventana Medical Systems, Inc.).

\section{Results}

Analysis of cervical cytology specimens by means of IMS resolved a pattern of peaks ranging from 2-10 kDa. Spectra from representative regions (regions of interest) of each sample were exported into ClinProTools and grouped into 


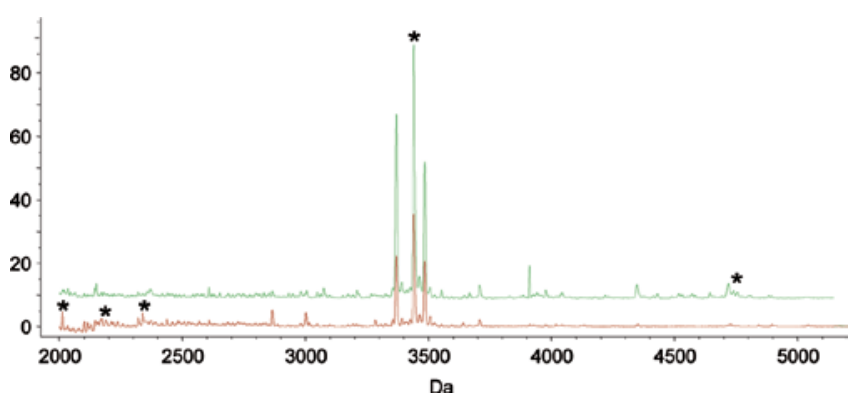

Figure 1. Overall sum spectra in the mass range of $2-5 \mathrm{kDa}(\mathrm{m} / \mathrm{z})$ obtained from the regions of interest of all 18 positive cytology specimens (red) and of all 14 negative cytology specimens (green), respectively. Asterisks indicate the five peaks used to generate the genetic algorithm-based model.

Table I. Algorithms for the classification of cytology samples.

\begin{tabular}{lcc}
\hline Algorithm & Sensitivity (\%) & Specificity (\%) \\
\hline GA & 88.9 & 78.6 \\
SVM & 88.9 & 71.4
\end{tabular}

Different algorithms to separate samples from patients with positive and negative cytology were generated using the ClinProTools 2.2 software. GA, genetic algorithm; SVM, support vector machine. the appropriate negative (Pap I-II) or positive (Pap $\geq$ IIID) class groupings to be used for model generation. Overall sum spectra of these regions of interest are demonstrated in Fig. 1, showing protein profiles from $2-5 \mathrm{kDa}$. To classify the positive and negative cytology samples successfully, two different models were generated with the software, which in turn can clearly distinguish between both groups (Table I). Utilizing a GA-based model positive and negative cytology samples could be discriminated with an overall cross-validation of $83.7 \%$ resulting in a sensitivity and a specificity of $88.9 \%$ and $78.6 \%$, respectively. This model is based on five peaks of different masses ranging from 2-4.7 kDa. Their molecular weight together with each peak's p-value and area under the curve (AUC) value are shown in Table II. Additionally, an SVM based model incorporating 14 peaks picked due to their differential expression in the overall sum spectra resulted in an overall cross-validation, sensitivity and specificity of $80.2 \%, 88.9 \%$ and $71.4 \%$, respectively. Three out of these 14 peaks were of special interest due to their higher impact on the classification and were also used in the GA-based model mentioned above.

By applying the generated GA-based model on all spectra obtained from representative cervical cytospin specimens we were able to group each spectrum of the sample into one of the two different classes. Those classification results can be visualized as so called class image. The color green was used
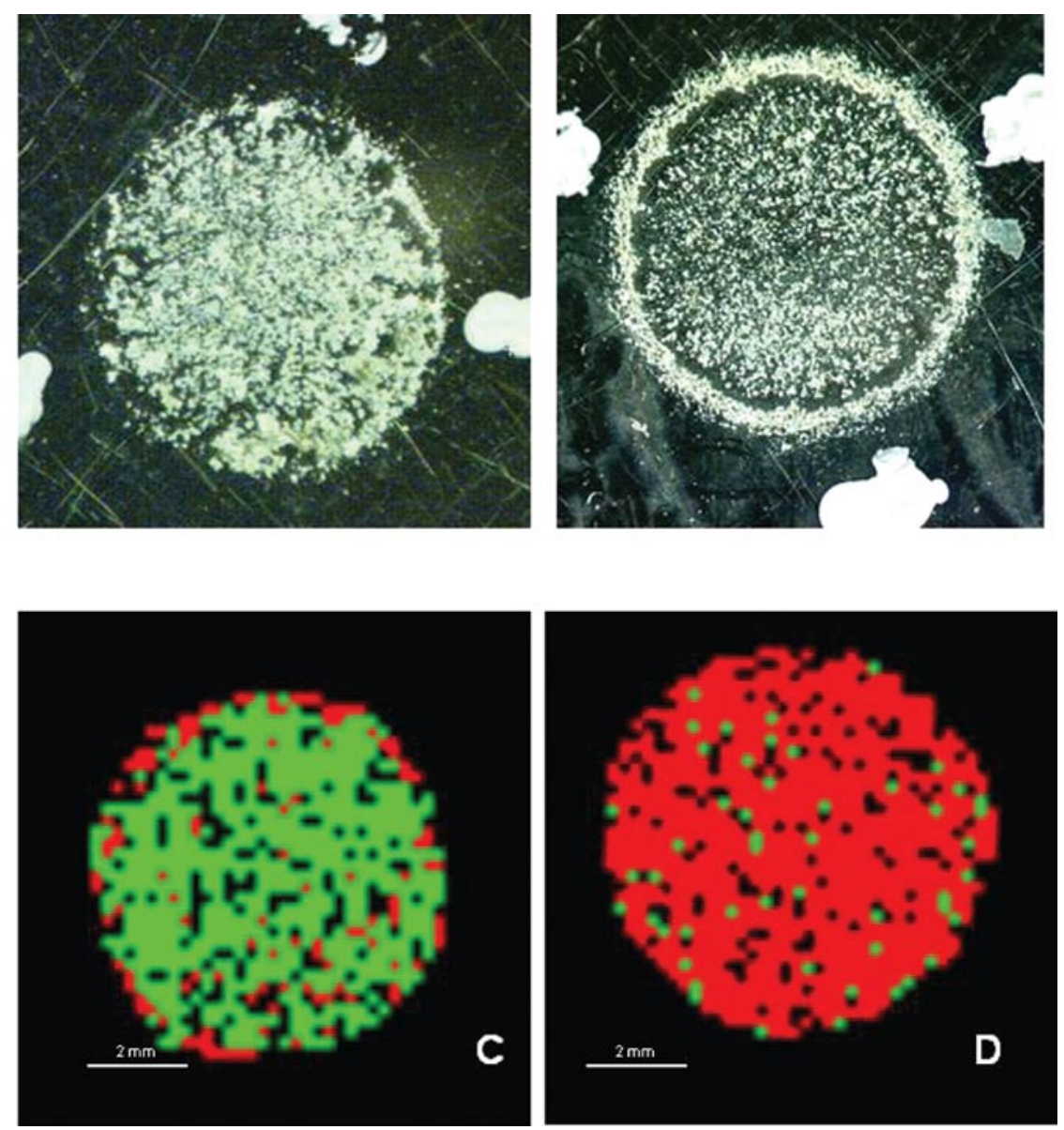

Figure 2. Representative images of unstained cytospin specimens of a negative (A) and a positive (B) Pap smear. Class images of the same cytospin samples: negative Pap smear (C) and positive Pap smear (D), respectively. Green, negative cytology (Pap I-II); red, positive cytology (Pap IIID-IV); non-colored, unclassified. White points (A and B) visible near cytopsins resemble teaching points with liquid white-out. 
Table II. Peak list of a genetic algorithm-based model.

\begin{tabular}{lcc}
\hline MW (m/z) & p-value & AUC \\
\hline 2012 & 0.032 & 0.76 \\
2172 & 0.005 & 0.80 \\
2339 & 0.017 & 0.79 \\
3441 & 0.076 & 0.74 \\
4740 & 0.004 & 0.80
\end{tabular}

Peaks incorporated in the genetic algorithm based model together with their p-values and AUC. MW, molecular weight; $\mathrm{m} / \mathrm{z}$, mass to charge ratio; AUC, area under the curve.

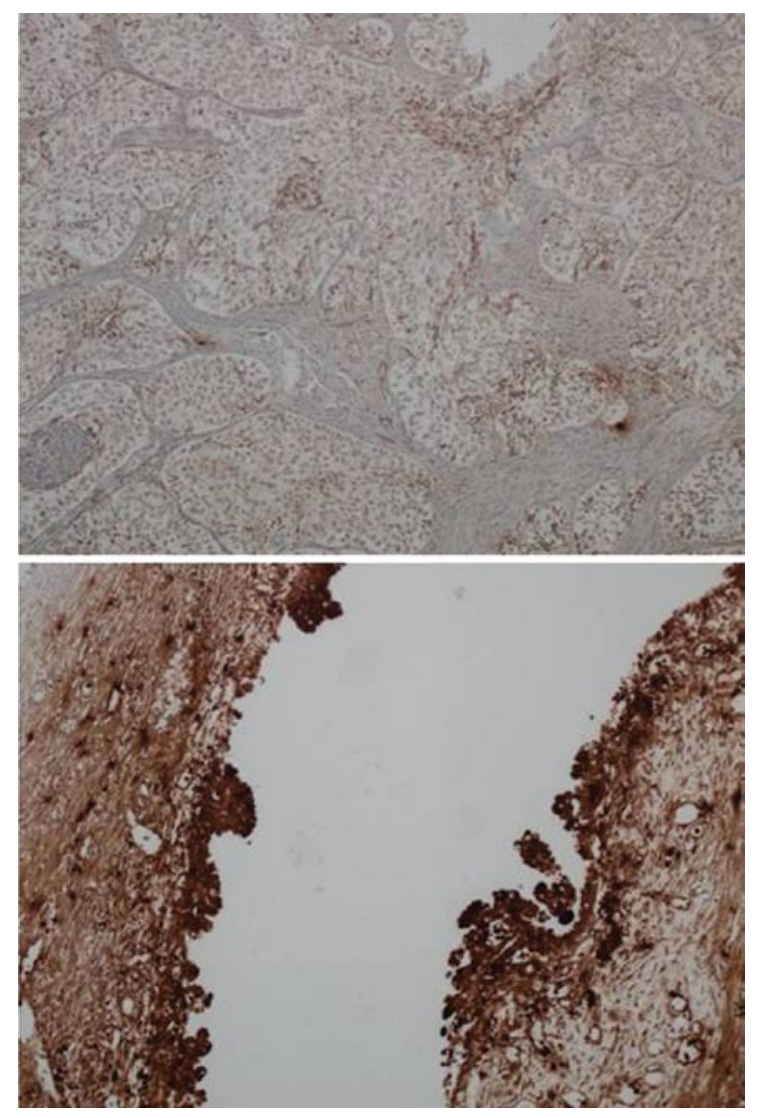

Figure 3. $\alpha$-defensin expression analyzed by immunohistochemistry. The top panel is an example of squamous cell carcinoma with no/reduced expression of $\alpha$-defensin; the bottom panel shows a representative image of a normal transitional zone of a uterus removed for leiomyoma with normal defensin expression.

for negative cytology and red for positive cytology. Pixels neither green nor red are due to unclassified spectra, meaning spectra belonging to none of the two above mentioned classes or spectra of non-sufficient quality, i.e. null spectra. Two representative cytospin specimens, one with a positive and one with a negative cervical cytology result, are shown in Fig. 2.

Three peaks of higher intensity in the negative cytology specimens $(\mathrm{m} / \mathrm{z}=3371,3441$, and 3485$)$ have previously been identified as $\alpha$-DEFA1, $\alpha$-DEFA2, and $\alpha$-DEFA3, respectively $(22,23)$. One of these peaks $(\mathrm{m} / \mathrm{z} 3441)$ is one of the classifiers in the GA and the SVM based model. DEFAs are a family of microbicidal and cytotoxic peptides involved in phagocytemediated host defense and are abundant in neutrophil granules (24). To validate these findings 10 uterine resection specimens were analyzed by immunohistochemistry to identify the source of DEFA expression. Normal squamous cells from the transition zone as well as neutrophils show a positive staining pattern whereas, SCC samples show little or no staining of the tumor cells and therefore, no protein expression (Fig. 3). Detailed data will be published elsewhere.

\section{Discussion}

Infection with HR types of HPV is the main causal factor of cervical cancer. Typically, cervical cancer develops over a long period of time with a variety of precursor lesions that can be detected primarily by cytological screening. However, the majority of these precursor lesions (i.e. squamous dysplasia) regress spontaneously. One major challenge of cancer screening is the ability of risk stratification; especially the ability to identify patients at risk of tumor progression. Since the currently applied screening procedure, the microscopic analysis of Pap smears, is a very time-consuming and subjective test with specificity and sensitivity below $100 \%$, there is a strong need for improvement.

Accordingly, existing screening procedures should be optimized and complemented by new techniques. Detection and identification of HR-HPV-DNA is a major component in risk stratification, however, its specificity is limited as it can be positive even in the absence of any cytological or genetic alteration of the cells. A variety of biomarkers (i.e. p16) and proliferation-associated proteins (i.e. TOP2A) are currently being investigated in international studies, but no definitive conclusions on their efficacy can yet be drawn.

Therefore, the present study was designed to investigate the usefulness of IMS as a tool to aid in triaging Pap smears in a rapid and unbiased manner. This approach allows for automated classification of routine Pap smears into reactive and dysplastic/HPV positive while maintaining the sample integrity. It still enables subsequent validation by standard cytological evaluation by an expert pathologist. By applying the generated GA-based model on all spectra obtained from representative cervical cytospin specimens we were able to group each spectrum into one of the two different classes. Additionally, an SVM-based model resulted in an overall cross validation, sensitivity and specificity of $80.2 \%, 88.9 \%$ and $71.4 \%$, respectively.

The results of this study elucidate that molecular imaging with MS technology has the potential to be used not only in cytologic screening but also for the identification of HPV infection in routine cytology specimens. This leads to a potential for risk management which might avoid unnecessary procedures and costs. Therefore, the next step will be to address identification of differentially-expressed proteins demonstrated in this study and to conduct a prospective study including Pap group III and IIID cases.

\section{Acknowledgements}

We are grateful for the excellent technical assistance from $\mathrm{N}$. Reulen and P. Jirak. 


\section{References}

1. Guidozzi F: Screening for cervical cancer. Obstet Gynecol Surv 4: 247-252, 1996.

2. Birley HD: Human papillomaviruses, cervical cancer and the developing world. Ann Trop Med Parasitol 5: 453-463, 1995.

3. Tkeshelashvili VT, Bokhman JV, Kuznetzov VV, Maximov SJ and Chkuaseli GT: Geographic peculiarities of endometrial and cervical cancer incidence in five continents (Review). Eur J Gynaecol Oncol 2: 89-94, 1993.

4. DeMay RM: Cytopathology of false negatives preceding cervical carcinoma. Am J Obstet Gynecol 175: 1110-1113, 1996.

5. Wright TC, Kurman RJ and Ferenczy A: Precancerous lesions of the cervix. In: Blaustein's Pathology of the Female Genital Tract. 4th edition. Kurman RJ (ed). Springer-Verlag, New York, pp229-232, 1994.

6. Wright TC, Sun XW and Koulos J: Comparison of management algorithms for the evaluation of women with low-grade cytologic abnormalities. Obstet Gynecol 2: 202-210, 1995.

7. Hatch KD, Schneider A and Abdel-Nour MW: An evaluation of human papillomavirus testing for intermediate- and high-risk types as triage before colposcopy. Am J Obstet Gynecol 172: $1150-1155,1995$.

8. zur Hausen H: Papillomavirus infections - a major cause of human cancers. Biochim Biophys Acta 1288: F55-F78, 1996.

9. zur Hausen H: Papillomaviruses in human cancers. Proc Assoc Am Physicians 111: 581-587, 1999.

10. Wolf JK and Ramirez PT: The molecular biology of cervical cancer. Cancer Invest 19: 621-629, 2001.

11. Solomon D, Schiffman M, Tarone R and the ALTS study group: Comparison of three management strategies for patients with atypical squamous cells of undetermined significance: baseline results from a randomized trial. J Natl Cancer Inst 93: 293-299, 2001.

12. Lesnikova I, Lidang M, Hamilton-Dutoit S and Koch J: p16 as a diagnostic marker of cervical neoplasia: a tissue microarray study of 796 archival specimens. Diagn Pathol 4: 22, 2009.

13. Wentzensen $\mathrm{N}$ and Klug SJ: Early detection of cervical carcinomas: finding an overall approach. Dtsch Arztebl Int 37: 617-622, 2008
14. Celis JE and Gromov P: Proteomics in translational cancer research: toward an integrated approach. Cancer Cell 3: 9-15, 2003.

15. Hanash S: Disease proteomics. Nature 422: 226-232, 2003.

16. Chaurand P, Sanders ME, Jensen RA and Caprioli RM: Proteomics in diagnostic pathology. Am J Pathol 165: 1057-1068, 2004.

17. Chaurand P, Stoeckli M and Caprioli RM: Direct profiling of proteins in biological tissue sections by MALDI mass spectrometry. Anal Chem 71: 5263-5270, 1999.

18. Stoeckli M, Chaurand P, Hallahan DE and Caprioli RM: Imaging mass spectrometry: a new technology for the analysis of protein expression in mammalian tissues. Nat Med 7: 493-496, 2001.

19. Caldwell RL and Caprioli RM: Tissue profiling by mass spectrometry. Mol Cell Proteomics 4: 394-401, 2005.

20. Seeley EH and Caprioli RM: Special feature: molecular imaging of proteins in tissues by mass spectrometry. Proc Natl Acad Sci USA 47: 18126-18131, 2008.

21. Bauer JA, Chakravarthy AB, Rosenbluth JM, Mi D, Seeley EH, De Matos Granja-Ingram N, Olivares MG, Kelley MC, Mayer IA, Meszoely IM, Means-Powell JA, Johnson KN, Tsai CJ, Ayers GD, Sanders ME, Schneider RJ, Formenti SC, Caprioli RM and Pietenpol JA: Identification of markers of taxane sensitivity using proteomic and genomic analyses of breast tumors from patients receiving neoadjuvant paclitaxel and radiation. Clin Cancer Res 2: 681-690, 2010.

22. Albrethsen J, Bogebo R, Gammeltoft S, Olsen J, Winther B and Raskov H: Upregulated expression of human neutrophil peptides 1, 2 and 3 (HNP 1-3) in colon cancer serum and tumours: a biomarker study. BMC Cancer 5: 8, 2005.

23. Roesch-Ely M, Nees M, Karsai S, et al: Proteomic analysis reveals successive aberrations in protein expression from healthy mucosa to invasive head and neck cancer. Oncogene 26: 54-64, 2007.

24. Ganz T: Defensins: antimicrobial peptides of innate immunity. Nat Rev Immunol 3: 710-720, 2003. 